\title{
Prospective evaluation of probabilistic dose-escalated IMRT in prostate cancer
}

\author{
Daniel Wegener ${ }^{1}$, Bernhard Berger ${ }^{2}$, Zhoulika Outtagarts ${ }^{1}$, Daniel Zips ${ }^{1,3}$, Frank Paulsen ${ }^{1}$, \\ Martin Bleif ${ }^{4}$, Daniela Thorwarth ${ }^{5}$, Markus Alber ${ }^{6}$, Oliver Dohm ${ }^{5}$, Arndt-Christian Müller ${ }^{1}$

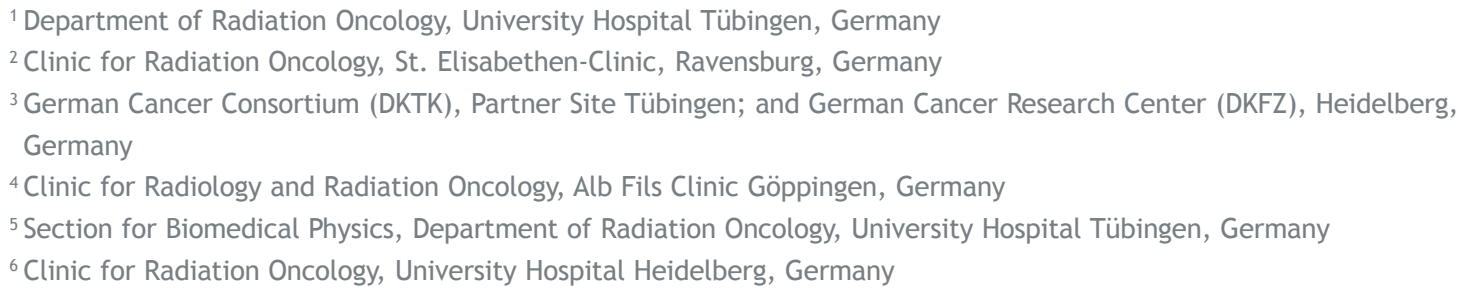

Radiol Oncol 2021; 55(1): 88-96.

Received 31 August 2020

Accepted 2 November 2020

Correspondence to: Daniel Wegener, Department of Radiation Oncology, University Hospital Tübingen, Eberhard Karls University Tübingen, Hoppe-Seyler-Str. 3, 72076 Tübingen. E-Mail: daniel.wegener@med.uni-tuebingen.de

Disclosure: No potential conflicts of interest were disclosed. We mention the cooperation with Siemens Healthcare, Philips, Elekta and PTB Braunschweig in another research project (DW, DZ, DT, ACM).

Background. Cure- and toxicity rates after intensity-modulated radiotherapy (IMRT) of prostate cancer are doseand volume dependent. We prospectively studied the potential for organ at risk (OAR) sparing and compensation of tumor movement with the coverage probability (CovP) concept.

Patients and methods. Twenty-eight prostate cancer patients (median age 70) with localized disease (cTlc-2c, NO, MO) and intermediate risk features (prostate-specific antigen [PSA] < 20, Gleason score $\leq 7 \mathrm{~b}$ ) were treated in a prospective study with the CovP concept. Planning-CTs were performed on three subsequent days to capture form changes and movement of prostate and OARs. The clinical target volume (CTV) prostate and the OARs (bladder and rectum) were contoured in each CT. The union of CTV1-3 was encompassed by an isotropic margin of $7 \mathrm{~mm}$ to define the internal target volume (ITV). Dose prescription/escalation depended on coverage of all CTVs within the ITV. IMRT was given in 39 fractions to 78 Gy using the Monte-Carlo algorithm. Short-term androgen deprivation was recommended and given in $78.6 \%$ of patients.

Results. Long-term toxicity was evaluated in 26/28 patients after a median follow-up of 7.1 years. At last follow-up, late bladder toxicity (Radiation Therapy Oncology Group, RTOG) G1 was observed in $14.3 \%$ of patients and late rectal toxicities (RTOG) of G1 (7.1\%) and of G2 (3.6\%) were observed. No higher graded toxicity occurred. After 7.1 years, biochemical control (biochemically no evidence of disease, bNED) was $95.5 \%$, prostate cancer-specific survival and the distant metastasis-free survival after 7.1 years were $100 \%$ each.

Conclusions. CovP-based IMRT was feasible in a clinical study. Dose escalation with the CovP concept was associated by a low rate of toxicity and a high efficacy regarding local and distant control.

Key words: probabilistic planned IMRT; coverage probability concept; prostate cancer; IMRT; dose escalation

\section{Background}

Cure- and toxicity rates after intensity modulated radiotherapy (IMRT) of prostate cancer are dose-dependent. ${ }^{1}$ Dose-escalated radiotherapy (RT) up to $80 \mathrm{~Gy}$ is a standard RT-technique ${ }^{2}$ and performed with (daily) image-guided RT (IGRT) combined with rapid treatment administration using volumetric modulated arc therapy (VMAT) or comparable techniques. ${ }^{2-4}$ Current improvements in regard to outcome and toxicity focus either on dose escalation or margin adaption. Dose escala- 
tion can be performed by using hypofractionated schedules of the whole prostate or of the dominant lesion alone ${ }^{5}$, Margin adaption i.e. reduction to reduce toxicity $+/$ - dose-escalation is performed by target tracking or (online) adaptive strategies. However, these techniques require not only modern RT instruments, which are not available yet for the majority of radiooncology departments, but also more time consumption per patient. $^{6}$

Also, planning target volume (PTV) margins still need to account for intra- and interfraction prostate motion to prevent target miss, while respecting the surrounding organs at risk (OAR), especially bladder and rectum. Therefore, alternative concepts which are applicable in width and which combine both dose escalation and toxicity reduction are of interest.

A promising concept to achieve a favorable PTVcoverage while individually sparing OARs represents Coverage probability based IMRT which does not need daily imaging due to its pre-adaptive planning approach. ${ }^{7-9}$ We studied the potential for Organ at Risk (OAR) sparing and compensation of tumor movement of the Coverage probability (CovP) concept within a prospective study and report 7-year outcome and toxicity rates.

\section{Patients and methods}

The coverage probability concept was investigated within a prospective study to evaluate in inter- mediate-risk prostate cancer patients' feasibility, toxicity and outcome parameter. The study was approved by the research board of the University Clinic of Tuebingen on 06/27/2007 (project-number: 257/2007B01).

\section{Patient enrollment}

Inclusion criteria were histopathologically confirmed adenocarcinoma of the prostate, intermediate-risk in D'Amico classification, age $\leq 85$ years at enrollment, Karnofsky-performance-status $\geq 70 \%$, localized disease (bone scan, abdominal/pelvic CT) and informed consent. Exclusion criteria were prior prostatectomy, prior transurethral resection (TURP) or previous irradiation of pelvis, a second malignancy or another severe, clinically prominent illness (e.g. decompensated heart insufficiency, chronic inflammatory bowel disease, blood coagulation restrictions etc.).

\section{Coverage probability concept}

A planning CT (pCT; SOMATOM Sensation 64, Siemens Healthcare, Erlangen) was performed daily over three days with a rectum- and bladder protocol in use. The pCT's were registered with Oncentra Masterplan $\AA$ (Theranostic GmbH, Solingen, Germany), then target volume and organ at risks (OAR's) were contoured in each pCT. The clinical target volume's (CTV) of each pCT (prostate $+1 \mathrm{~cm}$ seminal vesicle) were merged to create a CTVunion,
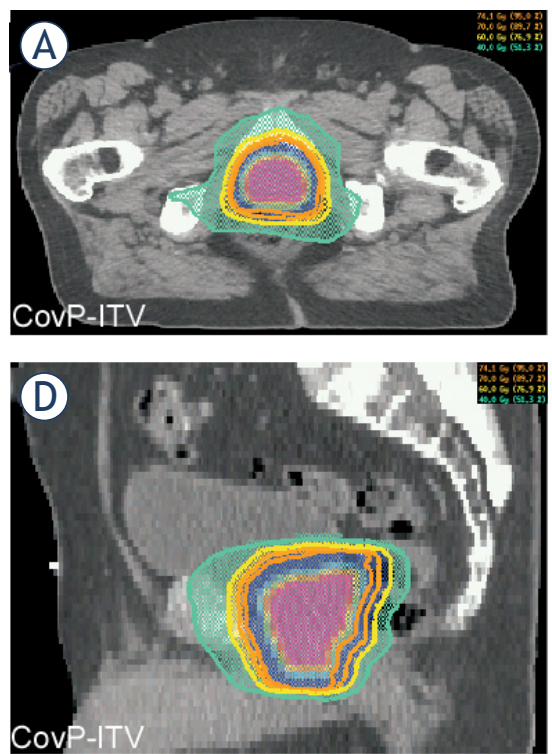
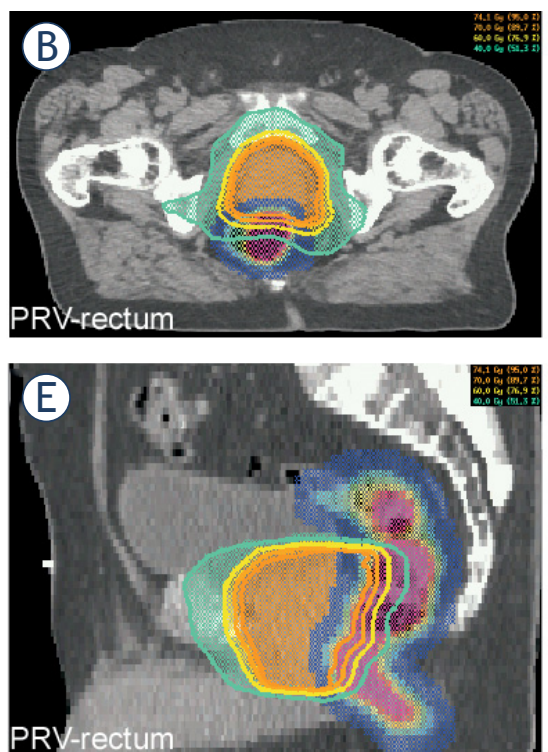
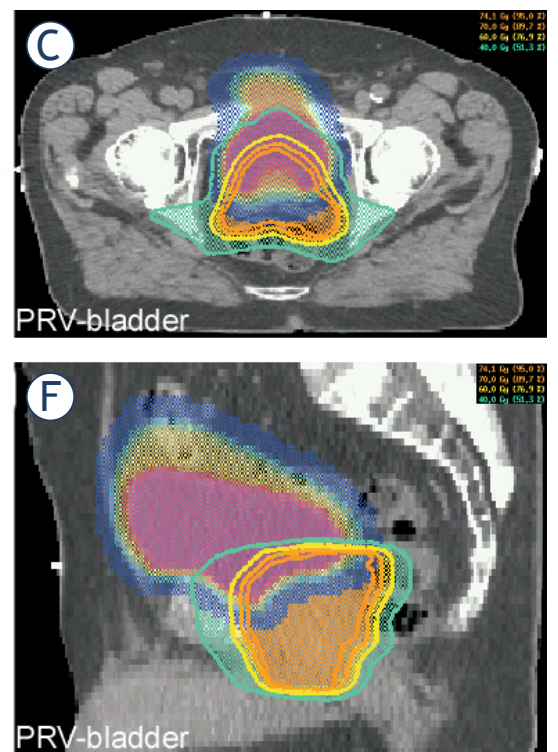

FIGURE 1. Coverage probability (CovP) concept: an example case is shown to indicate CovP-internal target volume (ITV) and planning organ at risk volumes (PRVs). 
TABLE 1. Patient characteristics of the study cohort

\begin{tabular}{llll}
\hline Parameter & $\begin{array}{l}\text { Mean (min.- } \\
\text { max.) }\end{array}$ & Median & $\mathrm{n}(\%)$ \\
\hline Age at treatment (years) & $70(56-83)$ & 70 & \\
Duration of radiotherapy (weeks) & $7.9(7.4-8.7)$ & 7.9 & \\
Gleason-Score (n, \%) & & & \\
6 & & $4(14.3 \%)$ \\
$7 a(3+4)$ & & $15(53.6 \%)$ \\
$7 b(4+3)$ & & $9(32.1 \%)$ \\
CTNM $(n, \%)$ & & \\
CT1c & & $8(28.6 \%)$ \\
CT2a & & $8(28.6 \%)$ \\
CT2b & & $3(10.7 \%)$ \\
CT2c & & $9(32.1 \%)$ \\
Neoadjuvant ADT (n, \%) & & $22(78.6 \%)$ \\
Duration of ADT (months, range) & $8.9(3.0-27.0)$ & 6.0 & \\
Initial PSA peak in ng/ml (range) & $9.1(1.9-19.8)$ & 8.3 & \\
PSA-peak by subgroup & & & $3(10.7 \%)$ \\
$0<x \leq 5$ & & & $15(53.6 \%)$ \\
$5<x \leq 10$ & & $8(28.6 \%)$ \\
$10<x \leq 15$ & & $2(7.1 \%)$ \\
$15<x \leq 20$ & & \\
\hline
\end{tabular}

$\mathrm{ADT}=$ androgen deprivation therapy; PSA = prostate-specific antigen

which was expanded by $7 \mathrm{~mm}$ to define an internal target volume (CovP-internal target volume [ITV]). The same margin of $7 \mathrm{~mm}$ was added to the merged OARs (rectum and bladder) to create planning OAR volumes (PRV). The coverage-probability approach uses spatially variable weight factors for "cost functions" for both ITV and OAR's. These weight factors are based on the probabilities of systematic organ shifts, estimated by the three planning CTs. For RT planning, "class solutions" were employed including OAR constraints, aimed target doses, optimal gantry angles as described elsewhere. ${ }^{7-9}$ (Figure 1).

\section{Treatment}

RT was performed as step-and-shoot IMRT with 78 Gy in 39 fractions. Planning software were Hyperion ${ }^{\circledR}$ versions 2.2.5, 2.2.6 and 2.3 (University Tuebingen, Germany; based on Monte Carlo algorithm). All patientsweretreated insupineposition on a 15 MV linear accelerator (Elekta Synergy S, Elekta Oncology Systems ${ }^{\circledR}$, Crawley, UK) equipped with a $4 \mathrm{~mm}$ multileaf collimator. Cone-beam CT (CBCT) was initially performed daily for three days and then the average positioning error was calculated and corrected for the next fraction. Thereafter, CBCT was routinely performed twice weekly to account for random positioning errors. In case of a positioning error of $>3 \mathrm{~mm}, \mathrm{CBCT}$ was repeated at the next RT treatment. Additional short term androgen deprivation therapy (ADT) for six months was recommended according to national guidelines. Further treatment specifications are given elsewhere.,10

\section{Patient follow-up and evaluation}

For each patient, baseline parameters for genitourinary (GU) and gastrointestinal (GI) functions were assessed and acute- and late toxicities were scored by a physician using common terminology criteria for adverse events (CTCAE $)^{10}$ and/or Radiation Therapy Oncology Group (RTOG) Classification. ${ }^{11}$ Initially, CTCAE version 2.0 was used, which was transferred in 2009 into version 4.0 for comparability. ${ }^{12}$ A physical examination, combined with scoring of late toxicity and measurement of features prostate-specific antigen (PSA)-level, was performed three months after RT and then once a year.

Primary outcome parameter was relapse-free survival (biological no evidence of disease, bNED). Biochemical relapse was defined by the Phoenixcriteria. ${ }^{13}$ Secondary outcome parameters were overall survival (OS), prostate-cancer specific survival (PCSS) and distant metastasis-free survival (DMFS). The outcome parameters were calculated from start of radiotherapy.

\section{Statistical analysis}

Descriptive statistics were performed with Microsoft Excel 2010 (Version 14.0.7145.5000). Further calculations, graphs and Kaplan-Meier estimations were performed with SPSS (Statistical Package for Social Sciences 'IBM SPSS Statistics for Windows, Version 24.0 (IBM Corp., Armonk, N.Y., USA)).

\section{Results}

\section{Patient characteristics and treatment}

Twentyeight patients were treated between 09/2008 and 10/2010 in this prospective study. Patient characteristics are given in Table 1. Dose escalation to 78 Gy was performed in all patients, the coverage probability concept was realized in 27/28 patients. In one patient, the study therapy was not accomplished due to unknown reasons (but a "standard" step-and shot IMRT to 78 Gy was performed based on one pCT). Neoadjuvant ADT was administered to $78.6 \%$ of patients with a mean duration of 8.9 months.

\section{Survival endpoints}

Median follow-up (FU) time was 7.4 years (range 0.7-9.3 years; mean 7.1 years). Median FU for PSAcontrol was 7.0 years (range 3.3-9.1 years; mean 6.8 years). PSA-nadir was reached on average after 2.7 
years (min.: 0.2 years; max.: 8.3 years; median: 1.3 years). Biochemical control is shown in Figure 2. Two patients developed a biochemical recurrence. PCSS and DMFS after 7 years were 100\% each. One patient died after a FU of 4.5 years due to a metastatic GIST which was detected 51 months after RT and located outside of the treated RT-field. Another patient was lost to FU.

\section{Acute toxicity}

Acute toxicity was defined as toxicity occurring from start of RT until the first FU-investigation $\leq 90$ days after end of RT. Weekly RTOG scoring results as well as maximum toxicity scores for GIand GU-toxicity are given in Supplement Figure 3. $46.4 \%$ of patients developed acute GU toxicity of maximum grade 1 and $42.9 \%$ developed acute GU toxicity of maximum grade 2 . No higher graded toxicity occurred. Acute GI toxicity of maximum grade 1 and 2 was found in $46.4 \%$ and $35.7 \%$ of pa-
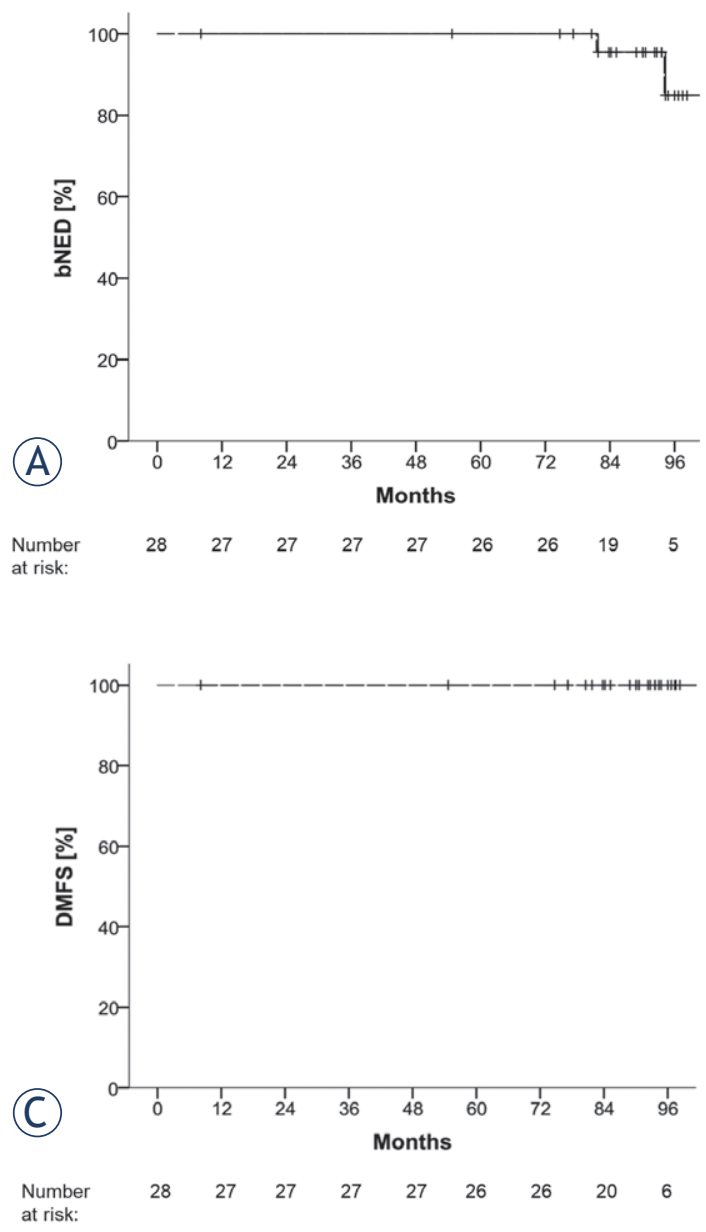

FIGURE 2. Survival outcomes: Kaplan-Meier curves of biochemically no evidence of disease (bNED), overall survival (OS), distant metastasis-free survival (DMFS) and prostate-cancer specific survival (PCSS). tients, respectively. No higher graded GI-toxicity occurred. Maximal GI- and GU-toxicity was seen in week 7 and 8 of radiotherapy. Additionally, CTCAE urinary incontinence was assessed: most patients $(n=22 ; 78.6 \%)$ did not develop incontinence. At first FU, two patients $(7.1 \%)$ reported grade 1 toxicity (no pads necessary) and no higher toxicity was reported. Skin erythema of RTOG grade 1 or 2 was present towards the end of radiotherapy in $78.6 \%(n=22)$ of patients and no higher graded skin toxicity occurred. At first FU, only one patient still suffered from RTOG grade 1 skin toxicity.

\section{Late toxicity}

Long-term toxicity was evaluated in $26 / 28$ patients after a median FU of 7.4 years (range $0.7-9.3$ years; mean 7.1 years). Toxicity was scored as cumulative maximum over study period and as toxicity at last follow-up (LFU).
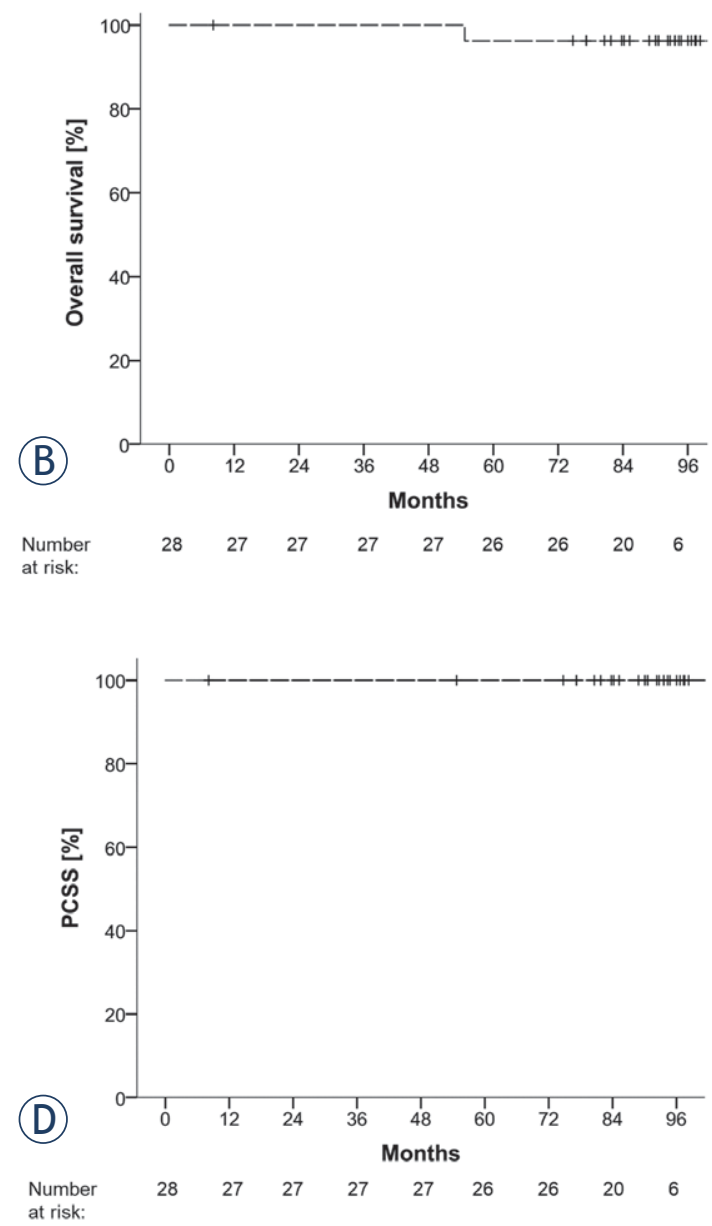


\section{Late GU-toxicity}

Most late GU-toxicity occurred 3-5 years post RT and seemed to decrease in prevalence towards LFU. Mild late bladder toxicity (RTOG G1) at LFU was observed in $14.3 \%$ of patients and no higher toxicity occurred (Figure 3). At LFU, 14.3\% and $10.7 \%$ of patients reported urinary frequency of CTC grades 1 and 2, respectively and no higher toxicity occurred. A urinary retention of CTC grade 1 and 2 was seen in $21.4 \%$ and $7.1 \%$ of patients at LFU (Figure 3). Urinary urgency of CTC grade 1 and 2 at LFU was present in $10.7 \%$ and $3.6 \%$ of patients showing no increase in prevalence over time. Urinary incontinence of CTC grade 1 (no pads needed) was reported by $21.4 \%$ of patients at LFU. Incontinence peaked 4-5 years after RT and then showed a decrease over time (supplement data).

\section{GI Toxicity}

Late rectal toxicities (RTOG) at LFU of grade 1 (7.1\% of patients) and of grade $2(3.6 \%)$ were observed (Figure 4). A peak in late GI-toxicity (RTOG) of grade 1 was seen 4 years post RT and then seemed to decrease again in prevalence. Gastrointestinal grade 3 toxicity, presenting with rectal bleeding, was observed in two patients (RTOG GI late, CTC rectal hemorrhage and CTC proctitis due to overlapping toxicity scoring). Both patients had pre-existing hemorrhoids and hemorrhoidal operations in their medical history. This occurred 9 months and 19 months post RT, respectively. After treatment with Argon-Plasma-Coagulation, no further grade 3 toxicity occurred in these two patients or others. Mild rectal hemorrhaging (CTC grade 1) was present in $14.3 \%$ of patients at LFU. Proctitis
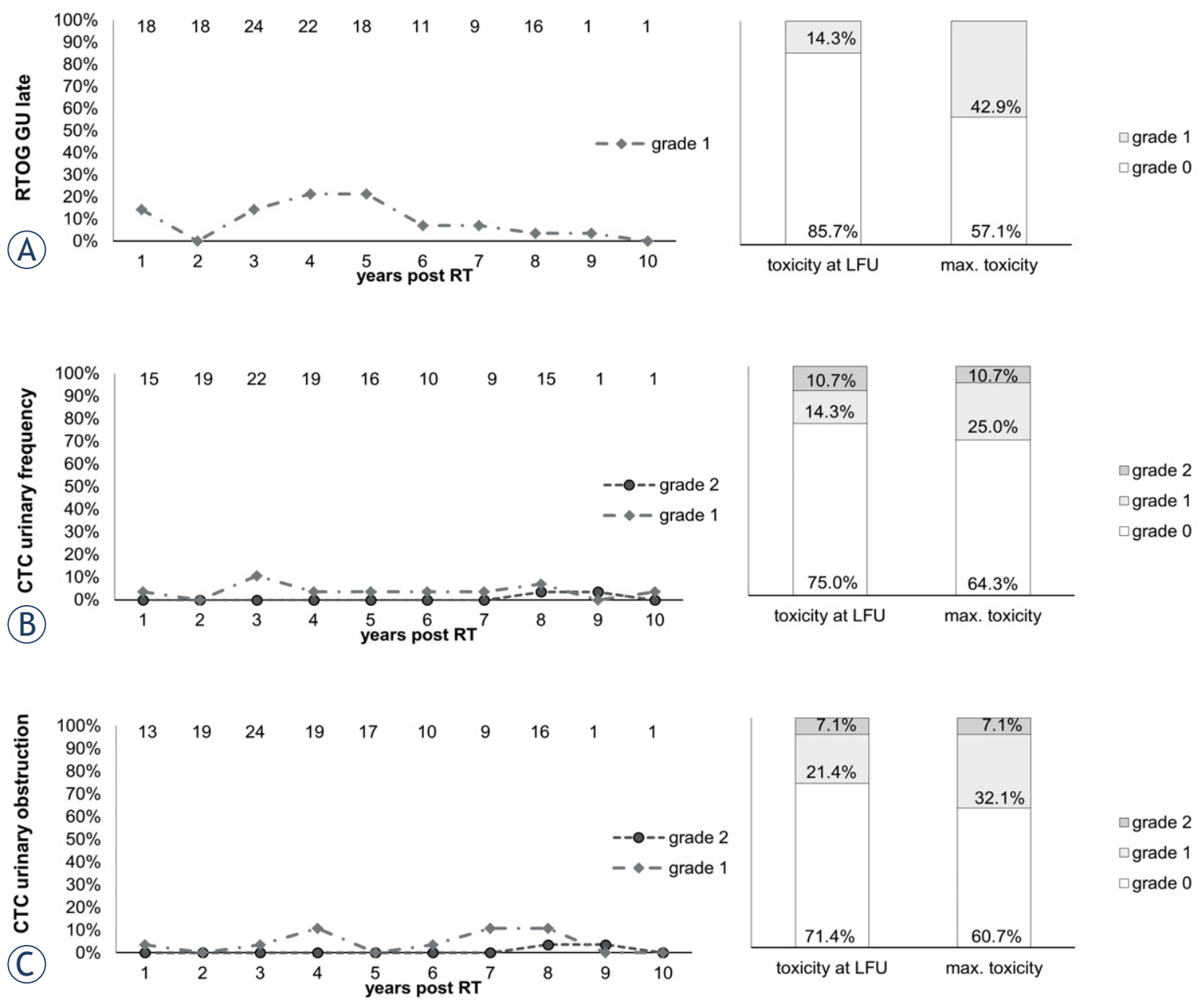

FIGURE 3. Late genitourinary (GU) toxicity items Radiation Therapy Oncology Group (RTOG) bladder late, common terminology criteria (CTC) urinary frequency and CTC urinary obstruction scored over the follow up (FU) period (left).

Y-axis: percentage of patients reporting the toxicity item. Top X-axis: number $n$ of patients with data available. Lower X-axis: years after radiotherapy. Right column: maximum late toxicity summed up by grade 
of CTC grade 1 (2) was present in $10.7 \%$ (7.1\%) of patients at LFU (Figure 4). Diarrhea of CTC grade 1 and 2 were present in $10.7 \%$ and $3.6 \%$ of patients, respectively. While diarrhea symptoms subsided 2 years post RT, it was reported again in one patient 8 years after radiotherapy (supplement data). Fecal incontinence of grade 1 and 2 occurred each in one patient $(3.6 \%)$, undulating over the FU period with no increase of severity of the symptom (supplement data).

\section{Dose-volume-histogram parameter}

Mean V70 PRV-bladder was $11.08 \%$ with this approach (accepted threshold: V70 bladder $<35 \%$ ). Mean V70 PRV-rectum was $13.03 \%$ (accepted threshold: V70 rectum $<15 \%)$. These constraints were based on the QUANTEC data and adjusted according to house standard. Due to these constraints, the mean CovP-ITV dose was 75.40 Gy $(\mathrm{V} 78=26.77 \%)$.

\section{Discussion}

In this prospective study, we report the feasibility and safety of a probabilistic planning concept for radiotherapy of intermediate risk prostate cancer patients based on the coverage probability. Doseescalation to 78 Gy was aimed with this technique showing very high long-term biochemical control rates and excellent survival rates. The toxicity profile was moderate with $85 \%$ of patients presenting without any GU/GI-toxicity at last follow-up in-
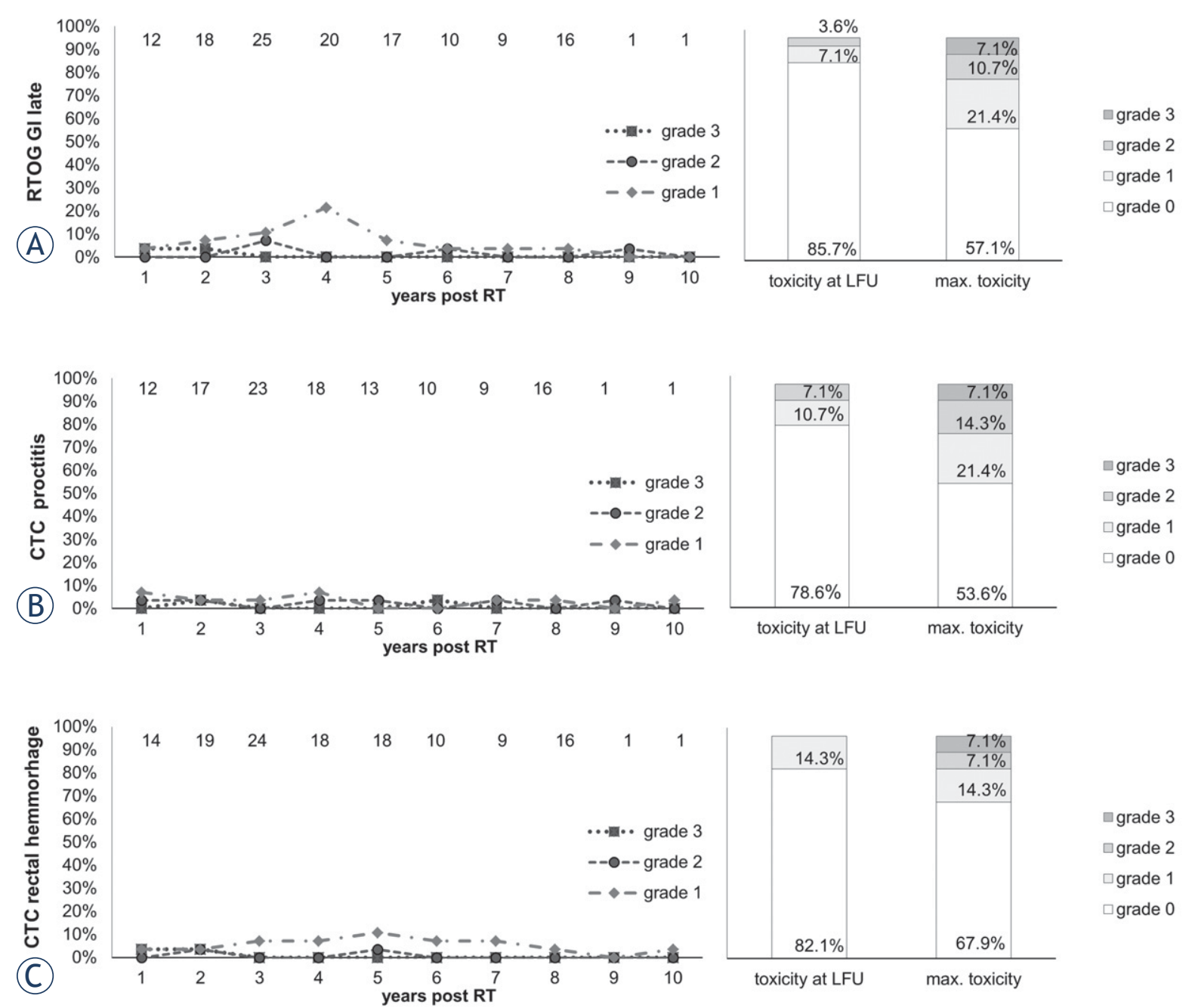

$\square$ grade 3

$\square$ grade 2

$\square$ grade 1

$\square$ grade 0

FIGURE 4. Late gastrointestinal (GI) toxicity items Radiation Therapy Oncology Group (RTOG) rectum late, common terminology criteria (CTC) proctitis and CTC rectal hemorrhage scored over the follow up (FU) period (left). 
dicating that $\operatorname{CovP}$ is a robust plan optimization achieving both low toxicity and favorable outcome.

In the planning phase of this study in 2007, dose escalation to 78 Gy was a novel concept in need of evaluation of safety and superiority to the former "standard" therapies of 3D-RT or IMRT of the prostate up to 70 Gy. Meanwhile, several studies could show a benefit to PCSS of dose escalation ${ }^{14-17}$ and a total dose of 74 to $80 \mathrm{~Gy}$ is the current standard of care and recommended in national guidelines..$^{2,3}$ Our study concept on probabilistic treatment planning is substantiated by excellent bNED-rates of $95.5 \%$ after 7.1 years (DMFS: $100 \%$, OS: $96.4 \%$ ). Two patients developed a biochemical recurrence. In one of these two patients, PSMA-PET-CT showed a local recurrence in the prostate. This patient had not received ADT due to cardiac diseases. In the other patient, a biochemical recurrence occurred at LFU and no further information on staging was available at the time of analysis of this study. Large studies of similar patient cohorts reached bNEDrates of $55-90 \%$ and DMFS of $88-100 \%$ (in the doseescalated study arms treated with normofractionated RT with 74-86.4 Gy total dose) after 5 years of FU. ${ }^{14-16,18-20}$ Compared to the 7.1 years FU of this study, only the M.D. Anderson trial could provide a longer FU period of 8.7 years in the mean. ${ }^{16}$ Our results are especially interesting in regard to the achieved mean ITV-dose of $75.40 \mathrm{~Gy}(\mathrm{~V} 78=26.7 \%)$ because this dose is in the lower dose range of the mentioned studies. Therefore, it could be hypothesized that robust treatment planning implementing probabilities of target volumes and OARs might be more relevant than dose-escalation to at least 78 Gy. And the standard one-CTV/PTV approach might be more at risk for target miss compared to a robust ITV concept, as was examined in another publication by our deparmenten. ${ }^{9}$ However, there are restrictions in comparability due to differences in the included tumor stages, ADT (no information about ADT in the study of Goldner et al.; no ADT in the M.D. Anderson trial. ${ }^{16,20}$ Taken together, the study treatment compares well to current standard treatments in PCSS and bNED.

The toxicity of this treatment approach is a central quality parameter, especially considering the potentially larger target volume (CovP-ITV) compared to a one-pCT approach. Acute GUand GI-toxicity was acceptable with only grade 1 and grade 2 (RTOG, CTC scores) and no grade 3+ toxicity. While certain items showed a peak after 3 weeks of RT (RTOG bladder acute) or 5 weeks (CTC urinary Incontinence, RTOG GI acute), most items showed an increase of toxicity prevalence towards the end of RT and the early peaks most likely origin in coincidental accumulation due to the limited patient size of the study. Towards the first FU-examination 3 months post RT, mostly grade 1 toxicity persisted. It is noteworthy that using the CTC-classification probably overestimates the item urinary incontinence in comparison to other studies since for example in the PROTECTstudy, incontinence of grade 1 was defined as need of $\geq 1$ pad per day which equals CTC incontinence of grade $2 .{ }^{21}$ In contrast, CTC incontinence of grade 1 - as measured in this study - is defined as occasional incontinence (pressure, coughing) not requiring pads.

Interestingly, late GU-toxicity of grade 1 or 2 (RTOG bladder late, CTC incontinence, CTC urgency) occurred only temporarily in this study population and decreased in prevalence after 5 years after RT. To date, consensus understanding of radiation biology is the continuous increase of late effects over time. ${ }^{22}$ No further data describing decreasing late toxicity after pelvic radiation is known to the authors. ${ }^{23,24}$ Due to the small sample size, random distribution of data and/or psychological factors such as patient habituation or recall bias cannot be ruled out.

Late GI-toxicity also showed no sign of increment over time and was generally rare: At LFU RTOG grade 2 toxicity was only present in one patient. Regarding rectal hemorrhaging, we suppose that the predisposition of hemorrhoids in those patients who developed this toxicity can be regarded as the (established) major risk factor. ${ }^{25}$ Taken together, the low toxicity rates in this cohort compare well to larger studies and demonstrate a favorable therapeutic window of the CovP-concept. ${ }^{14-16,18,20,23,26,27}$ The coverage concept has been described before in detail. $7,8,9$ The advantages include a safe margin with high PTV coverage, based on the individual OAR- and prostate motion of each patient, and measured by three planning-CTs. The idea behind the concept is a foreseen adaption of intra- and interfractional motion and deformation and therefore reduced verification intervals are needed. The efficacy of this method could be demonstrated in this study. Additionally, toxicity rates were not increased compared to standard techniques. The CovP-concept with $7 \mathrm{~mm}$ margin does not require daily image guidance due to its robustness as shown elsewhere. ${ }^{9}$ This might save radiation dose and on-table time for the department. Especially in patients who cannot undergo fiducial implementation or other invasive procedures such as rectum spacers $^{4,28}$ or patients with larger positional varia- 
tion of the prostate, this technique presents a useful and safe alternative. ${ }^{7}$

This study has several limitations: Further evaluation of outcome data in a larger study population is necessary. Additionally, there was no control group to compare toxicity and outcome to. In one patient, the study therapy was not accomplished due to unknown reasons. Three planningCTs are necessary for this method, causing extra burden on staff and patient, including additional radiation dose for the latter. However, we believe that the benefits, especially the reduced need for image verification or implantation of fiducials etc., outweigh the disadvantages and might even save radiation dose to the patient. Lastly, the performed step-and-shoot IMRT technique is mainly replaced by VMAT-techniques which leave slightly more options for OAR dose reduction and target dose escalation. ${ }^{9}$ However, the excellent late toxicity data and bNED rates support the relevance of this approach leading to an implementation in current study protocols. ${ }^{29}$

\section{Conclusions}

Coverage probability-based IMRT was feasible in a clinical study. Dose escalation with the CovPconcept was associated with a low rate of toxicity and a high efficacy regarding local and distant control, comparing well to studies with similar patient cohorts. Benefits include reduced target miss due to inclusion of potential inter- and intrafraction motion and reduced radiation dose due to less need for verification images. Utilization in other tumor entities and/or with contemporary RT techniques is being currently tested (NCT03617133).

\section{Availability of data and material and authors contributions}

The datasets used and/or analyzed during the current study are available from the corresponding author on reasonable request.

$\mathrm{BB}, \mathrm{MA}$. OD, FP and $\mathrm{MB}$ were responsible for the creation of the study protocol, ethical approval, patient enrollment and study therapy. DW, ZO and ACM were responsible for data acquisition, statistical analysis and writing of the manuscript. DT, DZ, FP and MA were responsible for manuscript revision and proof reading.

\section{References}

1. Viani GA, Stefano EJ, Afonso SL. Higher-than-conventional radiation doses in localized prostate cancer treatment: a meta-analysis of randomized, controlled trials. Int J Radiat Oncol Biol Phys 2009; 74: 1405-18. doi: 10.1016/j. ijrobp.2008.10.091

2. National Comprehensive Cancer Network I. NCCN Clinical Practice Guidelines in Oncology. Prostate cancer. 2018; Version 4.2018. August 15, 2018: 34.

3. Deutsche Krebsgesellschaft DK, AWMF. Leitlinienprogramm Onkologie. Interdisziplinäre leitlinie der qualität s3 zur früherkennung, diagnose und therapie der verschiedenen stadien des prostatakarzinoms 2018; Langversion 5.0:394.

4. Wegener D, Zips D, Thorwarth D, Weiß J, Othman AE, Grosse U, et al. Precision of T2 TSE MRI-CT-image fusions based on gold fiducials and repetitive T2 TSE MRI-MRI-fusions for adaptive igrt of prostate cancer by using phantom and patient data. Acta Oncol 2019; 58: 88-94. doi: 10.1080/0284186X.2018.1518594

5. Monninkhof EM, van Loon JWL, van Vulpen M, Kerkmeijer LGW, Pos FJ, Haustermans $K$, et al. Standard whole prostate gland radiotherapy with and without lesion boost in prostate cancer: toxicity in the flame randomized controlled trial. Radiother Oncol 2018; 127: 74-80. doi: 10.1016/j. radonc.2017.12.022

6. Schroeck FR, Jacobs BL, Bhayani SB, Nguyen PL, Penson D, Hu J. Cost of new technologies in prostate cancer treatment: systematic review of costs and cost effectiveness of robotic-assisted laparoscopic prostatectomy, intensitymodulated radiotherapy, and proton beam therapy. Eur Urol 2017; 72: 712-35. doi: 10.1016/j.eururo.2017.03.028

7. Baum C, Alber M, Birkner M, Nüsslin F. Robust treatment planning for intensity modulated radiotherapy of prostate cancer based on coverage probabilities. Radiother Oncol 2006; 78: 27-35. doi: 10.1016/j.radonc.2005.09.005

8. Baum C, Birkner M, Alber M, Paulsen F, Nüsslin F. Dosimetric consequences of the application of off-line setup error correction protocols and a hullvolume definition strategy for intensity modulated radiotherapy of prostate cancer. Radiother Oncol 2005; 76: 35-42. doi: 10.1016/j.radonc.2005.06.006

9. Outaggarts Z, Wegener D, Berger B, Zips D, Paulsen F, Bleif M, et al. Target miss using PTV-based IMRT compared to robust optimization via coverage probability concept in prostate cancer. Acta Oncol 2020; 59: 911-7. doi: 10.1080/0284186X.2020.1760349.

10. Trotti A, Colevas AD, Setser A, Rusch V, Jaques D, Budach V, et al. CTCAE v3.0: development of a comprehensive grading system for the adverse effects of cancer treatment. Semin Radiat Oncol 2003; 13: 176-81. doi: 10.1016/ S1053-4296(03)00031-6

11. Cox JD, Stetz J, Pajak TF. Toxicity criteria of the Radiation Therapy Oncology Group (RTOG) and the European Organization for Research and Treatment of Cancer (EORTC). Int I Radiat Oncol Biol Phys 1995; 31: 1341-6. doi: 10.1016/0360-3016(95)00060-C

12. National Cancer Institute USDOHAHS. Common terminology criteria for adverse events (CTCAE) Version 4.0. NIH Publication No 09-5410 2009: 1-196.

13. Roach M 3rd, Hanks G, Thames H Jr, Schellhammer P, Shipley WU, Sokol $\mathrm{GH}$, et al. Defining biochemical failure following radiotherapy with or without hormonal therapy in men with clinically localized prostate cancer: recommendations of the RTOG-ASTRO Phoenix Consensus Conference. Int J Radiat Oncol Biol Ph 2006; 65: 965-74. doi: 10.1016/j.ijrobp.2006.04.02

14. Dearnaley DP, Jovic G, Syndikus I, Khoo V, Cowan RA, Graham JD, et al. Escalated-dose versus control-dose conformal radiotherapy for prostate cancer: long-term results from the MRC RT01 randomised controlled trial. Lancet Oncol 2014; 15: 464-73. doi: 10.1016/S1470-2045(14)70040-3

15. Peeters ST, Heemsbergen WD, Koper PC, van Putten WL, Slot A, Dielwart $\mathrm{MF}$, et al. Dose-response in radiotherapy for localized prostate cancer: results of the Dutch multicenter randomized phase III trial comparing 68 Gy of radiotherapy with 78 Gy. J Clin Oncol 2006; 24: 1990-6. doi: 10.1200/ JCO.2005.05.2530

16. Kuban DA, Tucker SL, Dong L, Starkschall G, Huang EH, Cheung MR, et al. Long-term results of the M. D. Anderson randomized dose-escalation trial for prostate cancer. Int J Radiat Oncol Biol Phys 2008; 70: 67-74. doi: $10.1200 / J C O .2005 .05 .2530$ 
17. Hall MD, Schultheiss TE, Smith DD, Tseng BP, Wong JY. The impact of increasing dose on overall survival in prostate cancer. Radiat Oncol 2015; 10: 115 doi: 10.1186/s13014-015-0419-3

18. Zietman AL, Bae K, Slater JD, Shipley WU, Efstathiou JA, Coen JJ, et al. Randomized trial comparing conventional-dose with high-dose conformal radiation therapy in early-stage adenocarcinoma of the prostate: long-term results from Proton Radiation Oncology Group/American College of Radiology 95-09. J Clin Oncol 2010; 28: 1106-11. doi: 10.1200/ JCO.2009.25.8475

19. Zietman AL, DeSilvio ML, Slater JD, Rossi CJ Jr, Miller DW, Adams JA, et al. Comparison of conventional-dose vs high-dose conformal radiation therapy in clinically localized adenocarcinoma of the prostate: a randomized controlled trial. JAMA 2005; 294: 1233-9. doi: 10.1001/jama.294.10.1233

20. Goldner G, Dimopoulos J, Kirisits C, Pötter R. Moderate dose escalation in three-dimensional conformal localized prostate cancer radiotherapy: single-institutional experience in 398 patients comparing $66 \mathrm{~Gy}$ versus $70 \mathrm{~Gy}$ versus 74 Gy. Strahlenther Onkol 2009; 185: 438-45. doi: 10.1007/s000662009-2033-5.

21. Donovan JL, Hamdy FC, Lane JA, Mason M, Metcalfe C, Walsh E, et al. Patient-reported outcomes after monitoring, surgery, or radiotherapy for prostate cancer. N Engl J Med 2016; 375: 1425-37. doi: 10.1056/ NEJMoa1606221

22. Dorr W, Hendry JH. Consequential late effects in normal tissues. Radiother Oncol 2001; 61: 223-31. doi: 10.1016/s0167-8140(01)00429-7

23. Di Franco R, Borzillo V, Ravo V, Ametrano G, Cammarota F, Rossett $\mathrm{S}$, et al. Rectal/urinary toxicity after hypofractionated vs. conventional radiotherapy in high risk prostate cancer: systematic review and meta analysis. Eur Rev Med Pharmacol Sci 2017; 21: 3563-75. doi: 10.26355/eurrev_201708_13266

24. Carvalho IT, Baccaglini W, Claros OR, Chen FK, Kayano PP, Lemos GC, et al. Genitourinary and gastrointestinal toxicity among patients with localized prostate cancer treated with conventional versus moderately hypofractionated radiation therapy: systematic review and meta-analysis. Acta Oncol 2018; 57: 1003-10. doi: 10.1080/0284186X.2018.1478126

25. Valdagni R, Kattan MW, Rancati T, Yu C, Vavassori V, Fellin G, et al. Is it time to tailor the prediction of radio-induced toxicity in prostate cancer patients? Building the first set of nomograms for late rectal syndrome. Int J Radiat Oncol Biol Phys 2012; 82: 1957-66. doi: 10.1016/j.jjrobp.2011.03.028

26. Aluwini S, Pos F Schimmel E, Krol S, van der Toorn PP et al. Hypofractionated versus conventionally fractionated radiotherapy for patients with prostate cancer (hypro): late toxicity results from a randomised, non-inferiority, phase 3 trial. Lancet Oncol 2016; 17: 464-74. doi: 10.1016/S14702045(15)00567-7

27. Jolnerovski M, Salleron J, Beckendorf V, Peiffert D, Baumann AS, Bernier V, et al. Intensity-modulated radiation therapy from $70 \mathrm{~Gy}$ to $80 \mathrm{~Gy}$ in prostate cancer: six-year outcomes and predictors of late toxicity. Radiat Oncol 2017; 12: 99. doi: 10.1186/s13014-017-0839-3

28. Eckert F, Alloussi S, Paulsen F, Bamberg M, Zips D, Spillner $P$, et al Prospective evaluation of a hydrogel spacer for rectal separation in doseescalated intensity-modulated radiotherapy for clinically localized prostate cancer. BMC Cancer 2013; 13: 27. doi: 10.1186/1471-2407-13-27.

29. Ramlov A, Assenholt MS, Jensen MF, Grønborg C, Nout R, Alber M, et al. Clinical implementation of coverage probability planning for nodal boosting in locally advanced cervical cancer. Radiother Oncol 2017; 123: 158-63. doi: 10.1016/j.radonc.2017.01.015 